\title{
Thromboseprophylaxe für besondere Fälle
}

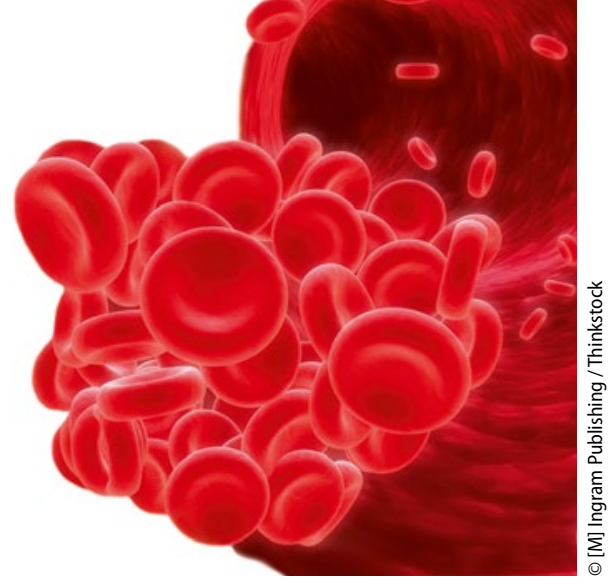

\author{
Patienten mit erhöhtem Thromboserisiko brauchen besondere diagnostische \\ Aufmerksamkeit und bei Bedarf die richtige Antikoagulation. Was dabei zu beachten \\ ist, erläutert Dr. P. Stiefelhagen am Beispiel von drei Patientengruppen.
}

? Eine Schwangerschaft bedeutet ja immer ein erhöhtes Risiko für eine tiefe Beinvenenthrombose bzw. eine Lungenembolie. Welche Diagnostik ist sinnvoll bzw. vertretbar, wenn ich bei einer Schwangeren den Verdacht auf eine Lungenembolie habe?

Da in der Schwangerschaft die D-Dimere oft positiv sind, beweist der Nachweis von D-Dimeren noch lange nicht, dass ein venöses thromboembolisches Ereignis vorliegt. Ein negatives Ergebnis schließt ein solches aber weitgehend aus. Bei einem positiven Ergebnis sollten Sie zunächst eine Kompressionssonografie durchführen oder veranlassen. Findet sich dabei eine tiefe Beinvenenthrombose, so ist die Diagnose gesichert und weitere Untersuchungen bzgl. Lungenembolie sind nicht erforderlich

Kann eine tiefe

Schwangere haben ein erhöhtes Thromboserisiko.

In der Tat, das Asthma bronchiale ist eine prothrombogene Situation. Warum das so ist, darüber gibt es unterschiedliche Meinungen. Einige glauben, dass es mit der Kortisontherapie zusammenhängt. Andere vertreten die Meinung, dass die dem Asthma zugrundeliegende Inflammation das Bindeglied zwischen beidem ist. Natürlich kann und soll man nicht bei jedem Asthmapatienten eine Thromboseprophylaxe durchführen.

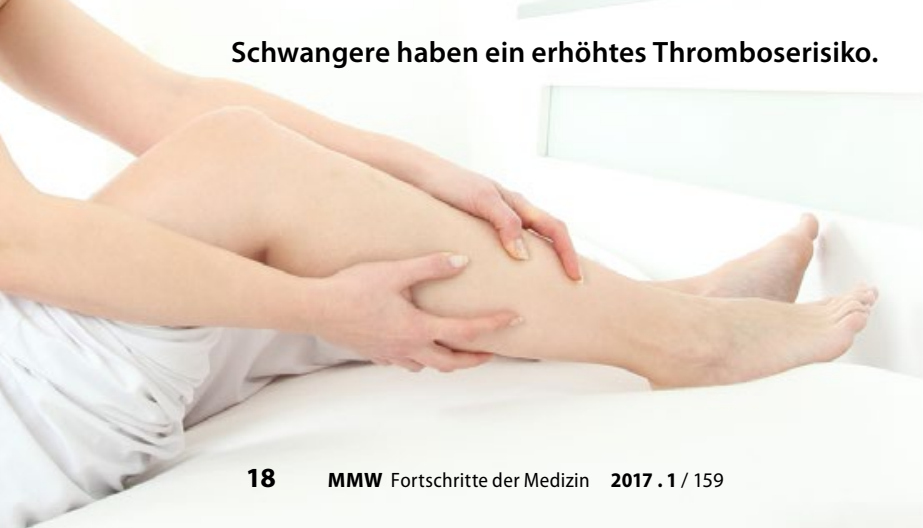

Kommen aber weitere Risiken wie beispielsweise eine vorübergehende Bettlägerigkeit bei einer schweren Exazerbation oder eine Superinfektion dazu, so ist eine solche sicherlich indiziert.

\section{? Darf ich bei Krebspatienten NOAKs zur Behandlung eines venösen thromboembolischen Ereig- nisses einsetzen?}

Bisher sind NOAKs bei dieser Indikation nicht offiziell zugelassen. Somit sind der jetzige Standard niedermolekulare Heparine (NMH). Auch aus haftungsrechtlichen Gründen sollte man NOAKs bei dieser Indikation deshalb nicht einsetzen. Allerdings gibt es immer mehr Studiendaten, die zeigen, dass ein NOAK bei Tumorpatienten genauso gut wirkt und genauso sicher ist wie bei Nicht-Tumorpatienten. Somit ist zu erwarten, dass NOAKs in absehbarer Zukunft die Zulassung auch für Tumorpatienten erhalten werden.

\section{? Tumorpatienten haben bekannt- lich ein deutlich erhöhtes thromboembolisches Risiko. Gilt dies auch für Patienten, die kurativ behan- delt sind?}

Die Daten einer aktuellen niederländischen Studie zeigen, dass das erhöhte Thromboserisiko sich weitgehend zurückbildet, wenn eine kurative Therapie erfolgreich war. Bei einem Krebsrezidiv steigt es aber wieder an.

Dr. med. Peter Stiefelhagen 